\title{
Distributed State Estimation for Dynamic Positioning Systems with Uncertain Disturbances and Transmission Time Delays
}

\author{
Li Liu $\mathbb{D}^{1,2}$ Tao Yao, ${ }^{1,2}$ Xin Hu, ${ }^{3}$ Chunjie Zhou, ${ }^{1,2}$ Dianli Hou, ${ }^{1}$ Shulin Feng, \\ and Hongyong Yang ${ }^{1}$ \\ ${ }^{1}$ Key Laboratory of Cyber-physical System and Intelligent Control in Universities of Shandong, \\ School of Information Science and Electrical Engineering, Ludong University, 264025 Yantai, Shandong, China \\ ${ }^{2}$ Yantai Research Institute of New Generation Information Technology, Southwest Jiaotong University, 264025 Yantai, China \\ ${ }^{3}$ School of Mathematics and Statistics, Ludong University, 264025 Yantai, Shandong, China
}

Correspondence should be addressed to Li Liu; liulildu@163.com

Received 20 May 2020; Accepted 23 June 2020; Published 20 July 2020

Guest Editor: Kailong Liu

Copyright $\odot 2020 \mathrm{Li}$ Liu et al. This is an open access article distributed under the Creative Commons Attribution License, which permits unrestricted use, distribution, and reproduction in any medium, provided the original work is properly cited.

The dynamic positioning system of unmanned underwater vehicles (UUVs) is a complex and large-scale system mainly due to the nonlinear dynamics, uncertainty in model parameters, and external disturbances. With the aid of the bio-inspired computing (BIC) method, the designed three-dimensional (3D) spatial positioning system is used for enlarging communication constraints and increasing signal coordination processing. With the growing of measurement scales, the issue of the networked high-precision positioning has been developed rapidly. Then, an information fusion estimation approach is presented for the distributed networked systems with data random transmission time delays and lost and disordered packets. To reduce the communication burden, an adaptive signal selection scheme is employed to reorganize the measurement sequence, and the parameter uncertainties as well as cross-correlated noise are used to describe the uncertain disturbances. Moreover, a reoptimal weighted fusion state estimation is designed to alleviate the information redundancy and maintain higher measurement accuracy. An illustrative example obtained from the $3 \mathrm{D}$ spatial positioning system is given to validate the effectiveness of the proposed method.

\section{Introduction}

For the complex and large-scale systems in the field of energy, transportation, logistics, and so on, due to the emerging problems such as strongly nonlinear and highly coupling, they significantly challenge the current computational tools. To solve science and engineering problems based on complex biological mechanisms and living phenomena, bio-inspired computing (BIC) method is used to design and develop the computer algorithms and models, such as neural networks [1], genetic algorithms [2], and artificial immune systems [3].

Taking the neural network as an example in the field of BIC, Tang et al. proposed a feedforward migration neural network to predict the battery aging trajectories, which arose from the highly nonlinear dynamics of battery aging [4]. In engineering application, the dynamic positioning system of unmanned underwater vehicles (UUVs) is a complex problem mainly due to the nonlinear dynamics, uncertainty in model parameters, and external disturbances. Using the adaptive method based on BIC computing is a promising solution to address these issues. Nevertheless, its full potential is yet to be realized, especially in underwater vehicle applications, mainly due to the highly nonlinear and timevarying nature of vehicle dynamics, unpredictable external disturbances, and the practical difficulty in accurately modeling the hydrodynamic effects [5]. In order to make the processing signal less sensitive to measurement noise, it suffers from scenarios such as the measurement information is inaccurate [6]. However, it is difficult to know the precise 
noise model in practice. Because of the absence of the full battery degradation model and the inevitable local aging fluctuations in the uncontrolled environments, a base model-oriented gradient-correction particle filter (GC-PF) [7] is proposed to predict aging trajectories of lithium-ion batteries. Therefore, as an estimation method that does not need to know the precise model of noise, such as $H_{\infty}$ filter, which considers the noise as random finite signals, has been developed in engineering applications like fault identification and estimation [6]. It should be noticed that both Kalman filter and $H_{\infty}$ filter are not suitable for nonlinear and non-Gaussian systems. In nonlinear systems, there are uncertainties caused by modeling errors and external disturbances.

Parameter uncertainties are inevitable in practical systems, and they may lead to severe performance degradation or even instability of the closed-loop system. Therefore, they should be fully considered in designing an estimator [8]. Liu et al. employed the Gaussian process regression (GPR) technique to capture the underlying mapping among capacity, storage temperature, and state-of-charge [9]. Moreover, two related data-driven models are developed based on GPR technique [10]. The uncertainties stem from uncertain hydrodynamic parameters, modeling errors, and unknown forces due to the ocean currents in an underwater environment. Specifically, the disturbance observer is developed to estimate the lumped disturbance composed of parametric model uncertainties, modeling errors, and unknown environmental forces. The command governor is formulated as a quadratically constrained quadratic programming problem [11]. Moreover, as opposed to the onestep prediction, multistep prediction is used herein, and a better performance can be achieved $[12,13]$.

As shown in Figure 1, the ship operating in the ocean inevitably suffers the disturbances due to waves, wind, and currents. In practice, the ship dynamics is related to the ship's own characteristics and the operating conditions; thus, there evidently exist parameter uncertainties in the ship motion mathematical model [14]. From Figure 1, it is believed that the concept of hybrid control can provide a scalable and stringent methodology for the design of real industrial control applications. A lot of research studies use the relevant BIC method to deal with several control objectives, changing environmental as well as operational conditions [15]. A ship's speed measurement before GPS is important to the navigation system. In real-time, closedloop control applications, there are problems such as noisy measurements and derivative processes. A variety of filtering methods are available to reduce the noise in the literature [16]. The Kalman filter is applied not only in signal processing but also in the estimation of velocity. The derivation of position signal data may be required not having another sensor for measuring velocity. A general derivative process is the Euler method, but this method may not give accurate results $[16,17]$.

Dynamic networks are interconnected dynamic systems with measured node signals and dynamic modules reflecting the links between the nodes. Various approaches have been developed for identification of dynamic networks, roughly

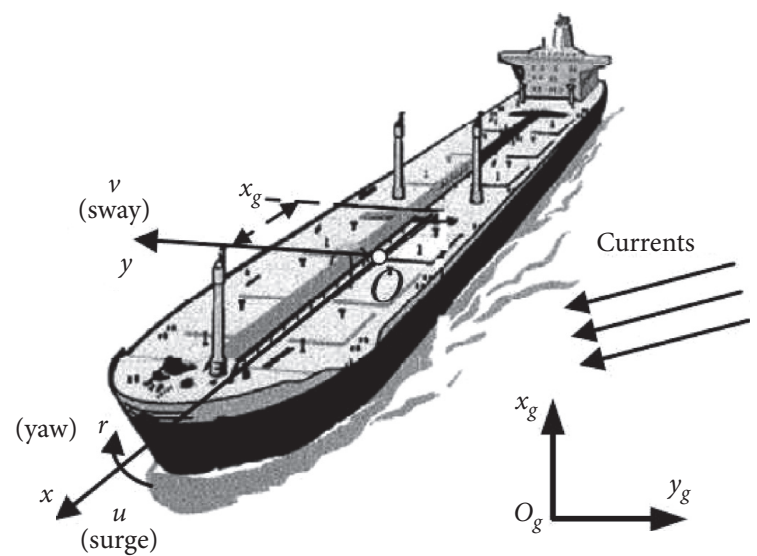

FIGURE 1: Frames and states of the ship.

divided into three categories. The first approach considers the identification of a single module in the dynamic network in the situation that the interconnection structure, or topology, of the network is known. The second approach focuses on identification of the full network dynamics for a given topology, and the last category deals with the identification of the topology (and dynamics) of the network [18]. Due to the influence of underwater observation conditions, positioning gross error will often appear; a method of adding the conditional constraints and confidence assessment to EKF was put forward to filter the positioning value, which can make the filtering result more robust and smooth [19]. Sato and Toda [20] proposed adaptive algorithms of gain tuning for Kalman filters and switching Kalman and $H_{\infty}$ filters for discrete systems. Both gain tuning and switching rely on square means of innovations. Accurate estimation of the horizontal component of the crane force is essential for the feedforward solution, but it is extremely difficult to obtain it from a measurement due to its time-varying nature and the nonlinear system dynamics stemming from the ship-environment interaction [21].

Considering the nonlinear and uncertain dynamic characteristics, a fuzzy neural network backstepping controller is designed to deal with the uncertainties, including estimation and compensation [22]. Brouwer et al. [23] presented an estimator to quantify the random uncertainty of the mean (RUM) from a stationary single time series, without performing actual repeat tests. To develop a robust ship dynamic modeling approach by making efforts on determining a common ship dynamic model and designing a robust identification method [24], the ship dynamic model should be suitable for cases which mean the sufficient balance between the complexity and the accuracy of the model.

Filtering and state estimation are important features of a dynamic positioning system, and estimates of the velocities must be computed from the noisy position and heading measurements through a state observer [25]. To solve the problem of high-precision measurement and positioning, the space measurement and positioning systems with relevant measurement methods have been developed rapidly. With the increasing of the network scale, the scope of the 
measurement and positioning systems is more and more large; meanwhile, the coverage mode is more and more flexible. Considering the complexity of the communication links, the energy and computing power of each node are limited. The configuration structure uses the strategy of all nodes, shares, coordinates, and communicates with the signal processing workloads. Accordingly, the network performance does not increase with the size of the network attenuation. Therefore, the use of the distributed network structure can have the ability to increase the reliability of information and improve the precision of collaborative calculation. Moreover, the distributed network structure reduces the burden of communication [26].

The distributed estimation strategy is applied to target tracking and positioning, fault diagnosis, and so on. The objective is that state estimation accuracy after fusion is higher than that of each local estimation. Research on filtering is the basis of the information perception and fusion; according to different performance indicators, relevant distributed filtering has aroused more and more attention, including distributed particle filtering [27], distributed $H_{\infty}$ filtering, and distributed Kalman filtering. Furthermore, distributed Kalman filtering is used for realizing the coordination and fusion of error estimation, which is an important scheme to solve the state estimation for large-scale systems [28]. A data-driven approach combining the RBF neural network (NN) and the extended Kalman filter (EKF) was proposed to estimate the internal temperature for lithium-ion battery thermal management $[29,30]$.

Due to the restriction of communication facilities and scale for networked systems, the measurement information is inevitably affected by the unpredictable interference. Note that the fundamental Kalman filtering method cannot satisfy the performance requirement such that the robust Kalman filtering method is designed considering various noise sequences. Costa and Benites [31] investigated robust pattern independent filtering for discrete-time Markov jump systems with state-correlated noises. Considering the correlation for noise, Feng et al. and Yan et al. [32,33] presented the distributed Kalman filtering fusion method with crosscorrelation between measurement noise and process noise. In addition, for a class of noisy sequences with uncertain variance, Keshavarz-Mohammadiyan and Khaloozadeh [34] used the method of solving the least upper bound of all admissible uncertainties for its true filtering error covariance matrix. The noise variance is set to be suitable in the current system state during the iterative process, and the estimation precision is improved. To deal with multiple time-delay systems and reduce the computational complexity, the received information may be asynchronous in the presence of transmission time delays during network communication. The method of measurement transformation developed the Kalman filter using the recombination of the innovation sequence. Due to restructuring the measurement sequence, the system with time delay is transformed into the correlative one with free time-delay [35].

In the engineering of the ship navigation applications, due to the complex issue of transmission over networks and external disturbances, the dynamic spatial positioning is difficult with the requirements of high-precision positioning and growing measurement errors. Motivated by the above analysis, based on the principle of spatial positioning of linear charge-coupled devices (CCD), the information measured by the three-dimensional (3D) photoelectric sensor is considered to be influenced by the uncertain factors, such as transmission time delays and cross-correlation noise, and the accuracy of state estimation is improved. In order to improve the robustness for the state estimation, the robust finite-horizon filtering is transformed into Kalman prediction to reduce the computational complexity using the method of reorganizing and transforming the measurement sequence as well as the innovation sequence. The proposed weighted fusion approach of filtering error covariance is used for coordinating and exchanging the measurement information between two subsystems. Therefore, based on the optimal information fusion criterion, the distributed weighted fusion estimation using an adaptive selection scheme is the reoptimization of the local estimation, which is able to obtain higher estimation precision than the local one.

The remainder of the paper is organized as follows. The dynamic spatial positioning system is modelled in Section 2. Section 3 presents the distributed weighted fusion method using an adaptive signal selection scheme. Simulation results and analysis for the dynamic spatial positioning system are presented in Section 4, and the concluding remarks are given in Section 5.

\section{Dynamic Positioning System Model}

The dynamic positioning system is designed by the 3D photoelectric sensor, and the principle of spatial positioning is shown in this section.

2.1. Principle of the 3D Photoelectric Sensor. Firstly, the Metris Krypton measurement approach based on the threecoordinate position measurement is developed. The $3 \mathrm{D}$ spatial position of each measuring point is obtained using the optical tracker and the special light emission device (LED). The measured target is made of a special LED, and its 3D coordinates are read from three sets of charge-coupled devices (CCD) on the optical tracker. Special LED placement can be moved arbitrarily, and it can be placed on any moving parts. And then, the processor is used for calculating the position change and direction from the LED at a certain time interval. As shown in Figure 2(a), the 3D photoelectric sensor is composed of three linear CCDs, which are embedded three fixed-focus cylindrical optical mirrors. The three linear CCDs have a " $Y$ " shape distribution on the same plane.

The positioning principle of the $3 \mathrm{D}$ photoelectric sensor is shown in Figure 2(b). The measured target is composed of the special LED. And then, each cylindrical optical mirror through light only projects to its corresponding plane under the CCD. A spatial point light source is projected into each CCD plane by a cylindrical optical mirror, which is straight line light intersecting with the CCD and is able to perceive its 


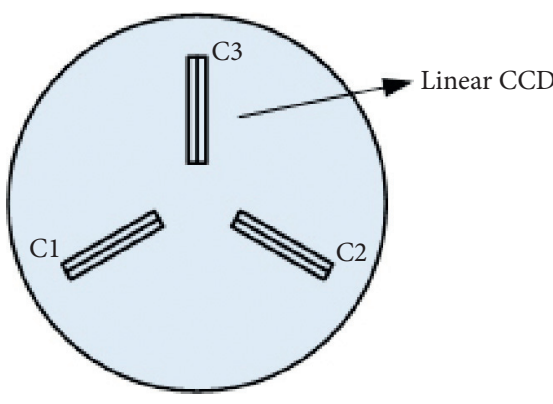

(a)

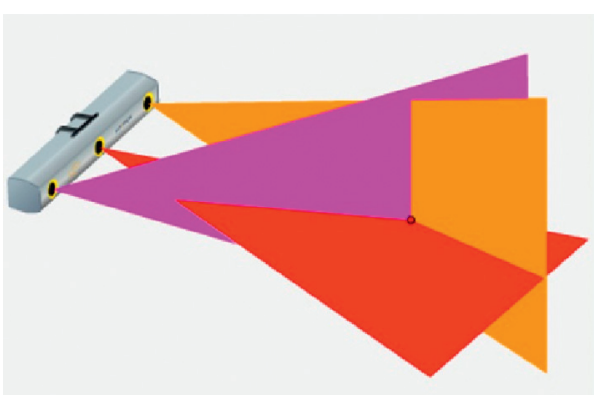

(b)

FIGURE 2: 3D photoelectric sensor. (a) Dynamic measurement device. (b) Spatial positioning principle.

position information on the CCD. If the targets are in the measurement range of the $3 \mathrm{D}$ photoelectric sensor, it will be decomposed into three planes through the three CCD lenses. Therefore, the processor is used to compute the intersection point for the three planes, that is, the coordinates of the light source for the space points $(X, Y, Z)$.

\subsection{System Model Based on the Adaptive Signal Selection} Scheme. Furthermore, considering the uncertainty of the distributed systems, the system model is established from the system state and measurement information, which is obtained by the sensor during the transmission process with uncertain disturbances and random transmission time delays.

More points in the space are measured by optical spatial positioning systems. It is necessary to use the fusion data from the coordination for multisensors. For the tracking system of the space motion target, the distributed configuration scheme is established, and the optimal estimation is designed to obtain more accurate measurement information. The considered system model for each sensor is described by the following linear discrete-time system $[36,37]$ :

$$
x(k+1)=\left(A_{k}+\mathscr{F}_{k} F_{k} E_{k}\right) x(k)+B_{k} w_{k}, \quad k=1,2, \ldots,
$$

$$
z^{i}(k)=\left(C_{k}^{i}+\mathscr{H}_{k}^{i} F_{k} E_{k}^{i}\right) x(k)+v_{k}^{i}, \quad i=1, \ldots, L .
$$

Note that $x(k) \in \mathbb{R}^{r}$ represents the system state, and $z^{i}(k) \in \mathbb{R}^{m_{i}}$ expresses the measurement for each sensor at time $k . A_{k} \in \mathbb{R}^{r \times r}, B_{k} \in \mathbb{R}^{r}, C_{k}^{i} \in \mathbb{R}^{m_{i} \times r}, \mathscr{F}_{k}, E_{k}, \mathscr{H}_{k}^{i}$, and $E_{k}^{i}$ are known time-varying matrices, and $F_{k}$ are the timevarying parameter uncertainties. $w_{k} \in \mathbb{R}$ and $v_{k}^{i} \in \mathbb{R}^{m_{i}}$ denote the process noise and measurement noise of the $i$-th sensor, which are zero-mean white noise with covariance matrices $Q_{k}$ and $R_{k}^{i}$, respectively. The initial state $x(0)$ with mean $\mu_{0}$ and covariance $P_{0}$ is assumed to be uncorrelated with other noise signals. Note that the uncertainties $F_{k}$ satisfy $F_{k} F_{k}^{T} \leq I$.

Due to the limited bandwidth, the transmission from sensors to processors inevitably generates the phenomenon of network congestion. To actively drop the disordered packets from the random transmission time delays, an adaptive signal selection scheme is designed (ASS).
Assumed that the measurement is sampled with a constant sampling period $T$, the sampling time instant is represented as $t \in\{k T, k \in \mathbb{N}\}$ for any subsystem. Set the maximum of the transmission delay as $N$, and it does not exceed the current time instant, i.e., $\tau(k) \leq k$. To further describe the networked system with random transmission time delays and lost and disordered packets, Figure 3 illustrates a typical scenario.

The current time instant $k$ receives a signal with timestamp $k_{1}$, and timestamp $k_{2}$ is expressed as the most recent signal before transmitting. From the sensor to the processor, the corresponding transmission time delays are recorded as $\tau\left(k_{1}\right) \in \mathbb{N}$ and $\tau\left(k_{2}\right) \in \mathbb{N}$, which denote the transmission time delays from the received packages using the ASS scheme or not at the sampling time $k$, which satisfies the following relationships:

$$
k=\tau\left(k_{1}\right)+k_{1}=\tau\left(k_{2}\right)+k_{2}
$$

Since the latest data packet during transmission is closer to the current data, a variable of delay $\beta(k) \geq 0$ is developed to express the relationship between $k_{1}$ and $k_{2}$, that is,

$$
k_{1}=k_{2}+\beta(k)
$$

From equations (3) and (4), $\tau\left(k_{1}\right)=\tau\left(k_{2}\right)-\beta(k)$ and $\tau\left(k_{1}\right) \leq \tau\left(k_{2}\right)$ are satisfied.

When receiving a valid data packet with timestamp using the ASS, in order to reduce the computational burden, the stored signal is reorganized as

$$
y_{\text {ASS }}(k)=z\left(k-\tau\left(k_{1}\right)\right) \text {. }
$$

Note that the data packet $y_{\text {ASS }}(k)$ is composed of the timestamp and transmission delay at each sampling time [38].

2.3. Uncertain Disturbances. To simplify the modeling of the wave and current for the UUV, considering unknown model parameters and uncertain time-varying disturbances in engineering applications, it is assumed that the bias model and the wave model are driven by zero-mean Gaussian noise. The offset of the vessel in surge, sway, and yaw is small as compared to the size of the ship. Suppose that the distributed systems involve the cross-correlation noise during networked transmission $[36,37,39]$. Meanwhile, the process 


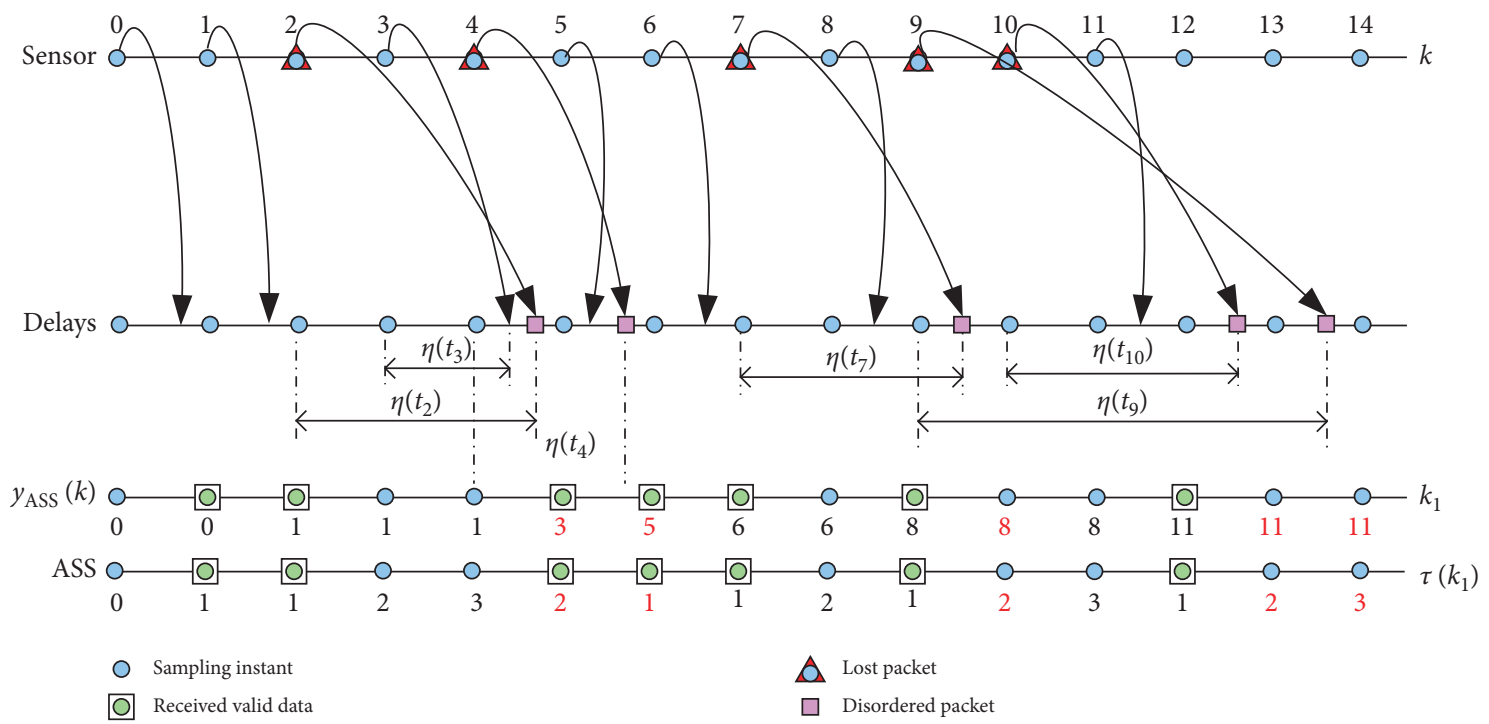

FIgURE 3: Process of packet sequence resorting using the ASS.

noise $w_{k}$ and measurement noise $v_{k}^{i}$ for each subsystem are interrelated at the same instant. Similarly, the measurement noise $v_{k}^{i}$ for the $i$-th subsystem and the measurement noise $v_{k}^{j}$ for the $j$-th subsystem are cross-related. The statistical attributes satisfy the following relationships:

$$
\begin{aligned}
& E\left(w_{k}\right)=0 \text {, } \\
& E\left(v_{k}^{i}\right)=0 \text {, }
\end{aligned}
$$

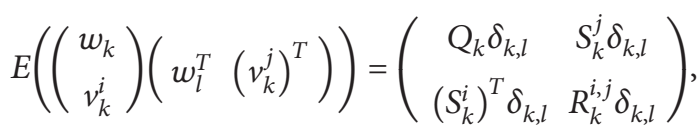

where the covariance satisfies $Q_{k}=Q_{k}{ }^{T}, R_{k}^{i, j}=\left(R_{k}^{j, i}\right)^{T}$, and $S_{k}^{i, j}=\left(S_{k}^{j, i}\right)^{T}$. Note that $\delta_{k, l}$ is the Kronecker function.

Since the stored measurement sequence contains random transmission time delays and lost and disordered packets, the distributed estimation problem is transformed into solving the optimal state estimation $\widehat{x}_{\text {ASS }}(k \mid k)$ using the ASS scheme, which fuses and compensates each local estimation $\hat{x}_{\text {ASS }}^{i}(t \mid t)$ with $t=k-\tau\left(k_{1}\right)$.

\section{ASS-Based Estimation}

For the considered distributed uncertain system in equations (1) and (2), a distributed state estimation approach is studied in this section based on robust finite-horizon filtering.

3.1. Reorganized Filter and Prediction Vector of the State. First of all, assume that the stored packet is $y_{\text {ASS }}^{i}(k)$ for the $i$ th subsystem at the current sampling time $k$. Depending on the role of the ASS, the received valid packet is $z^{i}\left(k-\tau\left(k_{1}\right)\right)$ with delay $\tau\left(k_{1}\right)$ before transmission. From equations (2) and (5), the reorganized measurement is denoted as

$$
y_{\text {ASS }}^{i}(k)=z^{i}(t)=\left(C_{t}^{i}+\mathscr{H}_{t}^{i} F_{t} E_{t}^{i}\right) x(t)+v_{t}^{i} .
$$

Based on the stored packet sequence $\left\{y_{\text {ASS }}^{i}(0), \ldots, y_{\text {ASS }}^{i}(k-1), y_{\text {ASS }}^{i}(k)\right\}$, the optimal state estimation $\hat{x}_{\text {ASS }}(k \mid k)$ with random transmission time delays can be obtained by the following local recursive method:

$$
\begin{gathered}
\hat{x}_{\mathrm{ASS}}^{i}(t \mid t)=\widehat{x}_{\mathrm{ASS}}^{i}(t \mid t-1)+K_{\mathrm{ASS}, t}^{i}\left(z^{i}(t)-\widehat{C}_{\mathrm{ASS}, t}^{i} \widehat{x}_{\mathrm{ASS}}^{i}(t \mid t-1)\right), \\
\widehat{x}_{\mathrm{ASS}}^{i}(t+1 \mid t)=\widehat{A}_{\mathrm{ASS}, t}^{i} \widehat{x}_{\mathrm{ASS}}^{i}(t \mid t-1)+L_{\mathrm{ASS}, t}^{i}\left(z^{i}(t)-\widehat{C}_{\mathrm{ASS}, t}^{i} \widehat{x}_{\mathrm{ASS}}^{i}(t \mid t-1)\right),
\end{gathered}
$$

in which $\hat{x}_{\mathrm{ASS}}^{i}(t \mid t)$ and $\hat{x}_{\mathrm{ASS}}^{i}(t+1 \mid t)$ represent the filter and the predictor, respectively, at the time instant $t$. Meanwhile, $\widehat{C}_{\mathrm{ASS}, t}^{i}, K_{\mathrm{ASS}, t}^{i}, \widehat{A}_{\mathrm{ASS}, t}^{i}$, and $L_{\mathrm{ASS}, t}^{i}$ are filtering parameters.

In order to solve the upper boundary for the filter and prediction covariance matrix, the augmented vectors of the state are defined as follows:

$$
\begin{aligned}
\Psi_{\mathrm{ASS}}^{i}(t) & =\left[\begin{array}{c}
e_{\mathrm{ASS}}^{i}(t) \\
\hat{x}_{\mathrm{ASS}}^{i}(t \mid t)
\end{array}\right], \\
\widetilde{\Psi}_{\mathrm{ASS}}^{i}(t+1) & =\left[\begin{array}{c}
\tilde{e}_{\mathrm{ASS}}^{i}(t+1) \\
\widehat{x}_{\mathrm{ASS}}^{i}(t+1 \mid t)
\end{array}\right],
\end{aligned}
$$

where $e_{\mathrm{ASS}}^{i}(t) \triangleq x(t)-\widehat{x}_{\mathrm{ASS}}^{i}(t \mid t)$ and $\widetilde{e}_{\mathrm{ASS}}^{i}(t+1) \triangleq x(t+1)-$ $\hat{x}_{\mathrm{ASS}}^{i}(t+1 \mid t)$. In addition, combining with the upper 
boundary, the augmented system modelled from equations (1) and (8)-(10) is rewritten as

$$
\Psi_{\mathrm{ASS}}^{i}(t)=\left(A_{\mathrm{ASS}, t 1}^{i}+H_{\mathrm{ASS}, t 1}^{i} F_{t} E_{\mathrm{ASS}, t 1}^{i}\right) \widetilde{\Psi}_{\mathrm{ASS}}^{i}(t)+D_{\mathrm{ASS}, t 1}^{i} v_{t}^{i},
$$

$$
\begin{aligned}
\widetilde{\Psi}_{\mathrm{ASS}}^{i}(t+1)= & \left(A_{\mathrm{ASS}, t 2}^{i}+H_{\mathrm{ASS}, t 2}^{i} F_{t} E_{\mathrm{ASS}, t 2}^{i}\right) \widetilde{\Psi}_{\mathrm{ASS}}^{i}(t) \\
& +B_{\mathrm{ASS}, t 2} w_{t}+D_{\mathrm{ASS}, t 2}^{i} v_{t}^{i} .
\end{aligned}
$$

The symbols are defined as

and

$$
\begin{aligned}
& A_{\mathrm{ASS}, t 1}^{i}=\left[\begin{array}{cc}
I-K_{\mathrm{ASS}, t}^{i} C_{t}^{i} & K_{\mathrm{ASS}, t}^{i}\left(\widehat{C}_{\mathrm{ASS}, t}^{i}-C_{t}^{i}\right) \\
K_{\mathrm{ASS}, t}^{i} C_{t}^{i} & I+K_{\mathrm{ASS}, t}^{i}\left(C_{t}^{i}-\widehat{C}_{\mathrm{ASS}, t}^{i}\right)
\end{array}\right], \\
& H_{\mathrm{ASS}, t 1}^{i}=\left[\begin{array}{c}
-K_{\mathrm{ASS}, t}^{i} \mathscr{H}_{t}^{i} \\
K_{\mathrm{ASS}, t}^{i} \mathscr{H}_{t}^{i}
\end{array}\right] \text {, } \\
& E_{\mathrm{ASS}, t 1}^{i}=E_{\mathrm{ASS}, t 2}^{i}=\left[\begin{array}{ll}
E_{t}^{i} & E_{t}^{i}
\end{array}\right] \text {, } \\
& D_{\mathrm{ASS}, t 1}^{i}=\left[\begin{array}{c}
-K_{\mathrm{ASS}, t}^{i} \\
K_{\mathrm{ASS}, t}^{i}
\end{array}\right] \text {, } \\
& H_{\mathrm{ASS}, t 2}^{i}=\left[\begin{array}{c}
\mathscr{F}_{t}^{i}-L_{\mathrm{ASS}, t}^{i} \mathscr{H}_{t}^{i} \\
L_{\mathrm{ASS}, t}^{i} \mathscr{H}_{t}^{i}
\end{array}\right] \text {, } \\
& A_{\mathrm{ASS}, t 2}^{i}=\left[\begin{array}{cc}
A_{t}-L_{\mathrm{ASS}, t}^{i} C_{t}^{i} & A_{t}-\widehat{A}_{\mathrm{ASS}, t}^{i}+L_{\mathrm{ASS}, t}^{i}\left(\widehat{C}_{\mathrm{ASS}, t}^{i}-C_{t}^{i}\right) \\
L_{\mathrm{ASS}, t}^{i} C_{t}^{i} & \widehat{A}_{\mathrm{ASS}, t}^{i}+L_{\mathrm{ASS}, t}^{i}\left(C_{t}^{i}-\widehat{C}_{\mathrm{ASS}, t}^{i}\right)
\end{array}\right], \\
& B_{\mathrm{ASS}, t 2}=\left[\begin{array}{c}
B_{t} \\
0
\end{array}\right] \text {, } \\
& D_{\mathrm{ASS}, t 2}^{i}=\left[\begin{array}{c}
-L_{\mathrm{ASS}, t}^{i} \\
L_{\mathrm{ASS}, t}^{i}
\end{array}\right] \text {. }
\end{aligned}
$$

Next, the covariance matrices are defined as $\widetilde{\Theta}_{\text {ASS }}^{i}(t)=$ $E\left[\Psi_{i}^{i}{ }_{\text {ASS }}(t)\left(\Psi_{\text {ASS }}^{i}(t)\right)^{T}\right]$ and $\widetilde{\Sigma}_{\text {ASS }}^{i}(t+1) \triangleq E\left[\widetilde{\Psi}_{\text {ASS }}^{i}(t+1)\right.$ $\left.\left(\widetilde{\Psi}_{\text {ASS }}^{i}(t+1)\right)^{T}\right]$ in equations (11) and (12). At the same time, the estimated error covariance matrices are derived from equations (11)-(15), and they are as follows:

$$
\begin{aligned}
\widetilde{\Theta}_{\mathrm{ASS}}^{i}(t)= & \left(A_{\mathrm{ASS}, t 1}^{i}+H_{\mathrm{ASS}, t 1}^{i} F_{t} E_{\mathrm{ASS}, t 1}^{i}\right) \widetilde{\Sigma}_{\mathrm{ASS}}^{i}(t)\left(A_{\mathrm{ASS}, t 1}^{i}+H_{\mathrm{ASS}, t 1}^{i} F_{t} E_{\mathrm{ASS}, t 1}^{i}\right)^{T}+D_{\mathrm{ASS}, t 1}^{i} R_{t}^{i}\left(D_{\mathrm{ASS}, t 1}^{i}\right)^{T}, \\
\widetilde{\Sigma}_{\mathrm{ASS}}^{i}(t+1)= & \left(A_{\mathrm{ASS}, t 2}^{i}+H_{\mathrm{ASS}, t 2}^{i} F_{t} E_{\mathrm{ASS}, t 2}^{i}\right) \widetilde{\Sigma}_{\mathrm{ASS}}^{i}(t) \times\left(A_{\mathrm{ASS}, t 2}^{i}+H_{\mathrm{ASS}, t 2}^{i} F_{t} E_{\mathrm{ASS}, t 2}^{i}\right)^{T}+B_{\mathrm{ASS}, t 2} Q_{t} B_{\mathrm{ASS}, t 2}^{T} \\
& +D_{\mathrm{ASS}, t 2}^{i} R_{t}^{i}\left(D_{\mathrm{ASS}, t 2}^{i}\right)^{T}+B_{\mathrm{ASS}, t 2} S_{t}^{i}\left(D_{\mathrm{ASS}, t 2}^{i}\right)^{T}+D_{\mathrm{ASS}, t 2}^{i}\left(S_{t}^{i}\right)^{T} B_{\mathrm{ASS}, t 2}^{T} .
\end{aligned}
$$

Finally, to find the upper boundary for the error covariance matrices, the filtering covariance is defined from the given equation (10):

$$
\widetilde{\Sigma}_{\mathrm{ASS}}^{i}(t)=\left[\begin{array}{cc}
\bar{\Sigma}_{\mathrm{ASS}}^{i}(t) & 0 \\
0 & P(t)-\bar{\Sigma}_{\mathrm{ASS}}^{i}(t)
\end{array}\right] .
$$

Note that the corresponding definitions of covariance matrices are $\bar{\Sigma}_{\text {ASS }}^{i}(t)=E\left[\widetilde{e}_{\text {ASS }}^{i}(t)\left(\tilde{e}_{\text {ASS }}^{i}(t)\right)^{T}\right]$ and $P(t)=E\left[x(t) x^{T}(t)\right]$.
3.2. Upper Boundary for Error Covariance. Based on the augmented vector of the state, the corresponding filter and prediction error covariance matrices are designed by Theorem 1 to detect the appropriate filtering parameters.

Theorem 1. For the unified form of $(A+H F E) X(A+H F E)^{T}$, if there is a positive scale $\alpha_{t}>0$ as well as a symmetrical positive matrix $\sum_{A S S}^{i}(t)$, they satisfy $\alpha_{t}^{-1} I-E_{\text {ASS } t 2}^{i} \Sigma_{\text {ASS }}^{i}(t)\left(E_{\text {ASS }, t 2}^{i}\right)_{i}^{T}>0$, and the inequality is established, i.e., $\Theta_{A S S}^{i}(t) \leq \Theta_{A S S}^{i}(t)$ and 
$\widetilde{\Sigma}_{\text {ASS }}^{i}(t+1) \leq \Sigma_{\text {ASS }}^{i}(t+1)$. Therefore, the upper boundaries for the error covariance matrices can be calculated by the following recursive formulas:

$$
\begin{aligned}
\Theta_{\mathrm{ASS}}^{i}(t)= & A_{\mathrm{ASS}, t 1}^{i} \Sigma_{\mathrm{ASS}}^{i}(t)\left(A_{\mathrm{ASS}, t 1}^{i}\right)^{T}+\alpha_{t}^{-1} H_{\mathrm{ASS}, t 1}^{i}\left(H_{\mathrm{ASS}, t 1}^{i}\right)^{T} \\
& +D_{\mathrm{ASS}, t 1}^{i} R_{t}^{i}\left(D_{\mathrm{ASS}, t 1}^{i}\right)^{T}+A_{\mathrm{ASS}, t 1}^{i} \Sigma_{\mathrm{ASS}}^{i}(t)\left(E_{\mathrm{ASS}, t 1}^{i}\right)^{T} \\
& \times\left(\alpha_{t}^{-1} I-E_{\mathrm{ASS}, t 1}^{i} \Sigma_{\mathrm{ASS}}^{i}(t)\left(E_{\mathrm{ASS}, t 1}^{i}\right)^{T}\right)^{-1} E_{\mathrm{AS}, t 1}^{i} \Sigma_{\mathrm{ASS}}^{i}(t)\left(A_{\mathrm{ASS}, t 1}^{i}\right)^{T} \\
\Sigma_{\mathrm{ASS}}^{i}(t+1)= & A_{\mathrm{ASS}, t 2}^{i} \Sigma_{\mathrm{ASS}}^{i}(t)\left(A_{\mathrm{ASS}, t 2}^{i}\right)^{T}+\alpha_{t}^{-1} H_{\mathrm{ASS}, t 2}^{i}\left(H_{\mathrm{ASS}, t 2}^{i}\right)^{T} \\
& +A_{\mathrm{ASS}, t 2}^{i} \Sigma_{\mathrm{ASS}}^{i}(t)\left(E_{\mathrm{ASS}, t 2}^{i}\right)^{T}\left(\alpha_{t}^{-1} I-E_{\mathrm{ASS}, t 2}^{i} \Sigma_{\mathrm{ASS}}^{i}(t)\left(E_{\mathrm{ASS}, t 2}^{i}\right)^{T}\right)^{-1} \\
& \times E_{\mathrm{ASS}, t 2}^{i} \Sigma_{\mathrm{ASS}}^{i}(t)\left(A_{\mathrm{ASS}, t 2}^{i}\right)^{T}+B_{\mathrm{ASS}, t 2}^{i} Q_{t}^{i}\left(B_{\mathrm{ASS}, t 2}^{i}\right)^{T} \\
& +D_{\mathrm{ASS}, t 2}^{i} R_{t}^{i}\left(D_{\mathrm{ASS}, t 2}^{i}\right)^{T}+B_{\mathrm{ASS}, t 2} S_{t}^{i}\left(D_{\mathrm{ASS}, t 2}^{i}\right)^{T}+D_{\mathrm{ASS}, t 2}^{i}\left(S_{t}^{i}\right)^{T} B_{\mathrm{ASS}, t 2}^{T} .
\end{aligned}
$$

Proof. The proof is similar to the derivation process in $[13,38]$.

And then, to probe the appropriate filtering parameters, the upper boundaries of the error covariance matrices are derived separately:

$$
\begin{aligned}
E\left[e_{\mathrm{ASS}}^{i}(t)\left(e_{\mathrm{ASS}}^{i}(t)\right)^{T}\right] & =\left[\begin{array}{ll}
I & 0
\end{array}\right] \widetilde{\Theta}_{\mathrm{ASS}}^{i}(t)\left[\begin{array}{l}
I \\
0
\end{array}\right] \\
& \leq\left[\begin{array}{ll}
I & 0
\end{array}\right] \Theta_{\mathrm{ASS}}^{i}(t)\left[\begin{array}{l}
I \\
0
\end{array}\right]=\bar{\Theta}_{\mathrm{ASS}}^{i}(t), \\
E\left[\widetilde{e}_{\mathrm{ASS}}^{i}(t+1)\left(\widetilde{e}_{\mathrm{ASS}}^{i}(t+1)\right)^{T}\right] & =\left[\begin{array}{ll}
I & 0
\end{array}\right] \widetilde{\Sigma}_{\mathrm{ASS}}^{i}(t+1)\left[\begin{array}{l}
I \\
0
\end{array}\right] \\
& \leq\left[\begin{array}{ll}
I & 0
\end{array}\right] \Sigma_{\mathrm{ASS}}^{i}(t+1)\left[\begin{array}{l}
I \\
0
\end{array}\right] \\
& =\bar{\Sigma}_{\mathrm{ASS}}^{i}(t+1) .
\end{aligned}
$$

Then, the optimal estimation based on the robust finitehorizon filtering in equations (8) and (9) is derived by the following Theorem 2.

Theorem 2. Suppose that the received valid measurement is $y_{A S S}^{i}(k)$ with $\tau\left(k_{1}\right)$-step transmission time delays at the current time $k$, and variable $\alpha_{t}$ is a positive scalar. Matrices $P(t)$ and $\bar{\Sigma}_{\text {ASS }}^{i}(t)$ are positive solutions, and they are derived from the following iteration formulas:

$$
\begin{aligned}
\bar{\Theta}_{\mathrm{ASS}}^{i}(t)= & \bar{\Sigma}_{\mathrm{ASS}}^{i}(t)+\bar{\Sigma}_{\mathrm{ASS}}^{i}(t)\left(E_{t}^{i}\right)^{T}\left(\widetilde{M}_{\mathrm{ASS}, t}^{i}\right)^{-1} E_{t}^{i} \bar{\Sigma}_{\mathrm{ASS}}^{i}(t) \\
& -\Lambda_{\mathrm{ASS}}^{i}(t)\left(\Xi_{\mathrm{ASS}}^{i}(t)\right)^{-1}\left(\Lambda_{\mathrm{ASS}}^{i}(t)\right)^{T}, \\
\bar{\Sigma}_{\mathrm{ASS}}^{i}(t+1)= & A_{t} \bar{\Sigma}_{\mathrm{ASS}}^{i}(t)\left(I+\left(E_{t}^{i}\right)^{T}\left(M_{\mathrm{ASS}, t}^{i}\right)^{-1} E_{t}^{i} \bar{\Sigma}_{\mathrm{ASS}}^{i}(t)\right) A_{t}^{T} \\
& -\Delta_{\mathrm{ASS}}^{i}(t)\left(\Xi_{\mathrm{ASS}}^{i}(t)\right)^{-1}\left(\Delta_{\mathrm{ASS}}^{i}(t)\right)^{T}+B_{t} Q_{t} B_{t}^{T}+\alpha_{t}^{-1} \mathscr{F}_{t} \mathscr{F}_{t}^{T}, \\
P(t+1)= & A_{t}\left(P^{-1}(t)-\alpha_{t}\left(E_{t}^{i}\right)^{T} E_{t}^{i}\right)^{-1} A_{t}^{T}+\alpha_{t}^{-1} \mathscr{F}_{t} \mathscr{F}_{t}^{T}+B_{t} Q_{t} B_{t}^{T},
\end{aligned}
$$

in which $\Lambda_{A S S}^{i}(t)=\bar{\Sigma}_{A S S}^{i}(t)\left(C_{t}^{i}\right)^{T}+\bar{\Sigma}_{A S S}^{i}(t)\left(E_{t}^{i}\right)^{T}\left(M_{A S S, t}^{i}\right)^{-1}$ $E_{t}^{i} \bar{\Sigma}_{A S S}^{i}(t)\left(C_{t}^{i}\right)^{T}$, and $\Delta_{A S S}^{i}(t)=A_{t} \bar{\Sigma}_{A S S}^{i} \quad(t)\left(I+\left(E_{t}^{i}\right)^{T}\right.$ $\left.\left(M_{\text {ASS, } t}^{i}\right)^{-1} E_{t}^{i} \bar{\Sigma}_{\text {ASS }}^{i}(t)\right)\left(C_{t}^{i}\right)^{T}+\alpha_{t}^{-1} \mathscr{F}_{t}\left(\mathscr{H}_{t}^{i}\right)^{T}+B_{t} S_{t}^{i}$. Note that the inequalities $P^{-1}(t)-\alpha_{t}\left(E_{t}^{i}\right)^{T} E_{t}^{i}>0$ and $M_{A S S, t}^{i}=\alpha_{t}^{-1} I-$ $E_{t}^{i} \bar{\Sigma}_{\text {ASS }}^{i}(t)\left(E_{t}^{i}\right)^{T}>0$ are satisfied.

Therefore, the results of the filtering parameters are solved as follows: 


$$
\begin{aligned}
\widehat{C}_{\mathrm{ASS}, t}^{i} & =C_{t}^{i}\left(I+\bar{\Sigma}_{\mathrm{ASS}}^{i}(t)\left(E_{t}^{i}\right)^{T}\left(M_{\mathrm{ASS}, t}^{i}\right)^{-1} E_{t}^{i}\right), \\
K_{\mathrm{ASS}, t}^{i} & =\Lambda_{\mathrm{ASS}}^{i}(t)\left(\Xi_{\mathrm{ASS}}^{i}(t)\right)^{-1}, \\
\widehat{A}_{\mathrm{ASS}, t}^{i} & =A_{t}\left(I+\bar{\Sigma}_{\mathrm{ASS}}^{i}(t)\left(E_{t}^{i}\right)^{T}\left(M_{\mathrm{ASS}, t}^{i}\right)^{-1} E_{t}^{i}\right), \\
L_{\mathrm{ASS}, t}^{i} & =\Delta_{\mathrm{ASS}}^{i}(t)\left(\Xi_{\mathrm{ASS}}^{i}(t)\right)^{-1} .
\end{aligned}
$$

From equation (22), we define the following symbols as $\Xi_{A S S}^{i}(t)=C_{t}^{i} \bar{\Sigma}_{\text {ASS }}^{i}(t)\left(I+\left(E_{t}^{i}\right)^{T}\left(M_{A S S, t}^{i}\right)^{-1} E_{t}^{i} \bar{\Sigma}_{\text {ASS }}^{i}(t)\right)\left(C_{t}^{i}\right)^{T}+$ $\alpha_{t}^{-1} \mathscr{H}_{t}^{i}\left(\mathscr{H}_{t}^{i}\right)^{T}+R_{t}^{i}$ and $\widetilde{M}_{A S S, t}^{i}=\alpha_{t}^{-1} I-E_{t}^{i} P(t)\left(E_{t}^{i}\right)^{T}$.

Proof. The upper boundary evolution is obtained by minimizing the estimation error covariance matrix, which is similar to solving the filtering parameters in $[12,13]$.

\subsection{State Estimation Based on Weighted Fusion Criteria.}

For each subsystem, taking into account the network-induced random transmission time delays and lost and disordered packets, the current estimated state is calculated by Theorem 2. In order to further improve the accuracy of state estimation, distributed weighted fusion estimation is studied in this section. Based on the weighted fusion criteria, the minimized estimation error cross-covariance matrix is used to perform the information exchange between any two subsystems.

Theorem 3. For a class of uncertain discrete time-varying systems, at the current sampling time $k$, the upper boundary for the filter error cross-covariance matrix $\bar{\Theta}_{\text {ASS }}^{l, j}(t)$ between the $i$-th subsystem and the j-th subsystem as well as the prediction error cross-covariance matrix $\bar{\Sigma}_{A S S}^{i, j}(t+1)$ are expressed as follows:

$$
\begin{aligned}
& \bar{\Theta}_{\mathrm{ASS}}^{i, j}(t)=\left[\begin{array}{ll}
I & 0
\end{array}\right] \Theta_{\mathrm{ASS}}^{i, j}(t)\left[\begin{array}{l}
I \\
0
\end{array}\right] \\
& =\left(I-K_{\mathrm{ASS}, t}^{i} C_{t}^{i}\right) \bar{\Sigma}_{\mathrm{ASS}}^{i, j}(t)\left(I-K_{\mathrm{ASS}, t}^{j} C_{t}^{j}\right)^{T} \\
& +\left(K_{\mathrm{ASS}, t}^{i}\left(\widehat{C}_{\mathrm{ASS}, t}^{i}-C_{t}^{i}\right)\right)\left(P(t)-\bar{\Sigma}_{\mathrm{ASS}}^{i, j}(t)\right)\left(K_{\mathrm{ASS}, t}^{j}\left(\widehat{C}_{\mathrm{ASS}, t}^{j}-C_{t}^{j}\right)\right)^{T} \\
& +\left(\bar{\Sigma}_{\mathrm{ASS}}^{i, j}(t)+K_{\mathrm{ASS}, t}^{i}\left(\widehat{C}_{\mathrm{ASS}, t}^{i}\left(P(t)-\bar{\Sigma}_{\mathrm{ASS}}^{i, j}(t)\right)-C_{t}^{i} P(t)\right)\right)\left(E_{t}^{i}\right)^{T}\left(\widetilde{M}_{\mathrm{ASS}, t}^{i, j}\right)^{-1} E_{t}^{j} \\
& \times\left(\bar{\Sigma}_{\mathrm{ASS}}^{i, j}(t)+K_{\mathrm{ASS}, t}^{j}\left(\widehat{C}_{\mathrm{ASS}, t}^{j}\left(P(t)-\bar{\Sigma}_{\mathrm{ASS}}^{i, j}(t)\right)-C_{t}^{j} P(t)\right)\right)^{T} \\
& +\alpha_{t}^{-1} K_{\mathrm{ASS}, t}^{i} \mathscr{H}_{t}^{i}\left(\mathscr{H}_{t}^{j}\right)^{T}\left(K_{\mathrm{ASS}, t}^{j}\right)^{T}+K_{\mathrm{ASS}, t}^{i} R_{t}^{i, j}\left(K_{\mathrm{ASS}, t}^{j}\right)^{T} \text {, } \\
& \bar{\Sigma}_{\mathrm{ASS}}^{i, j}(t+1)=\left[\begin{array}{ll}
I & 0
\end{array}\right] \Sigma_{\mathrm{ASS}}^{i, j}(t+1)\left[\begin{array}{l}
I \\
0
\end{array}\right] \\
& =\left(A_{t}-L_{\mathrm{ASS}, t}^{i} C_{t}^{i}\right) \bar{\Sigma}_{\mathrm{ASS}}^{i, j}(t)\left(A_{t}-L_{\mathrm{ASS}, t}^{j} C_{t}^{j}\right)^{T}+B_{t} Q_{t} B_{t}^{T}+L_{\mathrm{ASS}, t}^{i} R_{t}^{i, j}\left(L_{\mathrm{ASS}, t}^{j}\right)^{T} \\
& +\left(A_{t}-\widehat{A}_{\mathrm{ASS}, t}^{i}+L_{\mathrm{ASS}, t}^{i}\left(\widehat{C}_{\mathrm{ASS}, t}^{i}-C_{t}^{i}\right)\right)\left(P(t)-\bar{\Sigma}_{\mathrm{ASS}}^{i, j}(t)\right) \\
& \times\left(A_{t}-\widehat{A}_{\mathrm{ASS}, t}^{j}+L_{\mathrm{ASS}, t}^{j}\left(\widehat{C}_{\mathrm{ASS}, t}^{j}-C_{t}^{j}\right)\right)^{T} \\
& +\left(\left(A_{t}-L_{\mathrm{ASS}, t}^{i} C_{t}^{i}\right) \bar{\Sigma}_{\mathrm{ASS}}^{i, j}(t)+\left(A_{t}-\widehat{A}_{\mathrm{ASS}, t}^{i}+L_{\mathrm{ASS}, t}^{i}\left(\widehat{C}_{\mathrm{ASS}, t}^{i}-C_{t}^{i}\right)\right)\right. \\
& \left.\times\left(P(t)-\bar{\Sigma}_{\mathrm{ASS}}^{i, j}(t)\right)\right)\left(E_{t}^{i}\right)^{T}\left(\tilde{M}_{\mathrm{ASS}, t}^{i, j}\right)^{-1} E_{t}^{j}\left(\bar{\Sigma}_{\mathrm{ASS}}^{i, j}(t)\left(A_{t}-L_{\mathrm{ASS}, t}^{j} C_{t}^{j}\right)^{T}\right. \\
& \left.+\left(P(t)-\bar{\Sigma}_{\mathrm{LZ}}^{i, j}(t)\right)\left(A_{t}-\widehat{A}_{\mathrm{LZ}, t}^{j}+L_{\mathrm{LZ}, t}^{j}\left(\widehat{C}_{\mathrm{LZ}, t}^{j}-C_{t}^{j}\right)\right)^{T}\right) \\
& +\alpha_{t}^{-1}\left(\mathscr{F}_{t}-L_{\mathrm{LZ}, t}^{i} \mathscr{H}_{t}^{i}\right)\left(\mathscr{F}_{t}-L_{\mathrm{LZ}, t}^{j} \mathscr{H}_{t}^{j}\right)^{T}-B_{t} S_{t}^{j}\left(L_{\mathrm{LZ}, t}^{j}\right)^{T}-L_{\mathrm{LZ}, t}^{i}\left(S_{t}^{i}\right)^{T} B_{t}^{T},
\end{aligned}
$$
in $_{M_{A S S, t}^{i, j}}=\alpha_{t}^{-1} I-E_{t}^{i} \bar{\Sigma}_{A S S}^{i, j}(t)\left(E_{t}^{j}\right)^{T}$.
$\tilde{M}_{A S S, t}^{i, j}=\alpha_{t}^{-1} I-E_{t}^{i} P(t)\left(E_{t}^{j}\right)^{T}$

and

Proof. Its derivation process is similar to the solution of Theorem 2.
The optimal weighted fusion estimation can be used to solve the trace of the estimation error by minimizing the cross-covariance matrix $[33,40]$. Set $\hat{x}_{\text {ASS }}^{i}(k \mid t)$ as a local estimation with the $r$-dimensional random vector $x(k)$, which contains the network-induced factor. The distributed 
fusion estimator $\hat{x}_{\text {ASS }}(k \mid k)$ is designed as a $r$-dimensional vector:

$$
\widehat{x}_{\mathrm{ASS}}(k \mid k)=\Omega_{k}^{1} \hat{x}_{\mathrm{ASS}}^{1}(k \mid k)+\cdots+\Omega_{k}^{L} \widehat{x}_{\mathrm{ASS}}^{L}(k \mid k) .
$$

It is worth noting that solving $\widehat{x}_{\mathrm{ASS}}^{i}(k \mid k)$ from $\hat{x}_{\mathrm{ASS}}^{i}(k \mid t)$ develops the one-step prediction method to guarantee the precision of the estimation [35, 41].

Thus, the distributed weighted fusion estimation performance is optimal only if the weighted matrix given by equation (25) meets the following criteria [37, 41], i.e.,

$$
\left[\Omega_{k}^{1}, \ldots, \Omega_{k}^{L}\right]=\left(I_{0}^{T} \Pi_{\mathrm{ASS}}^{-1}(k) I_{0}\right)^{-1} I_{0}^{T} \Pi_{\mathrm{ASS}}^{-1}(k),
$$

in which $I_{0}=\underbrace{\left[I_{r}, \ldots, I_{r}\right]}_{L}$ is a $r L \times r$-dimensional matrix, and $\sum_{i=1}^{L} \Omega_{k}^{i}=I_{r}$. In equation (26), $\Pi_{\mathrm{ASS}}(k)$ is the symmetrical positive matrix, which is composed of $\Pi_{\mathrm{ASS}}^{i, j}(k)=E\left[e_{\mathrm{ASS}}^{i}(t)\left(e_{\mathrm{ASS}}^{j}(t)\right)^{T}\right], i=1, \ldots, L ; j=1, \ldots, L$.

Meanwhile, the corresponding optimal information fusion estimation of the cross-covariance is calculated by

$$
\begin{aligned}
\widetilde{\Pi}(k \mid k) & =E\left[(x(k)-\widehat{x}(k \mid k))(x(k)-\widehat{x}(k \mid k))^{T}\right] \\
& =\left(I_{0}^{T} \Pi_{\text {ASS }}^{-1}(k) I_{0}\right)^{-1} .
\end{aligned}
$$

Note that when $i=j, \widetilde{\Pi}(k \mid k) \leq \Pi_{\mathrm{ASS}}^{i}(k) \leq \bar{\Pi}_{\mathrm{ASS}}^{i}(k)$ is satisfied for the $i$-th subsystem.

So far, the solution process of the distributed weighted fusion estimation method based on the robust finite-horizon filtering using the ASS scheme is shown in Algorithm 1.

Remark 1. The distributed uncertain systems propose the ASS scheme to deal with the network-induced random transmission time delays and lost and disordered packet phenomena. As mentioned before, for the random transmission delay, the one-step prediction method is used to compensate the missing data packets. On the contrary, in order to realize the information exchange, the distributed state estimation based on the weighted fusion criteria is studied using the linear minimum covariance matrix to obtain the consistent expression of the system state. As a theoretical basis, the fusion scheme has higher estimation accuracy than each local subsystem.

\section{Numerical Simulations}

4.1. Simulation of Spatial Positioning. The spatial position structure of the 3D photoelectric sensor is shown in Figure 2(a). Set the length of the three equivalent linear CCDs as $8.8 \mathrm{~mm}$, which are embedded in the 3D photoelectric sensor. The focal length of the column mirror is $f=20 \mathrm{~mm}$, and the distance from the inner end of each CCD to the center of the coordinates is set as $10 \mathrm{~mm}$. The simulation results are demonstrated as follows.

4.1.1. Each Intersection of the Projection Line on the CCD. It is assumed that a spatial luminous point $P 0(x 0, y 0, z 0)$ is used as the spatial positioning point, and the measurable range is $4000 * 4000 * 6000 \mathrm{~mm}^{3}$ by the $3 \mathrm{D}$ photoelectric sensor. $P 0(x 0, y 0, z 0)$ passes through each focal point. i.e., $f 1, f 2, f 3$. And then, the light is divergent from the spatial luminous point. Note that the light is dispersed into a straight line through a cylindrical prism based on the refraction of light principle. The three CCDs project onto the $X-Y$ plane, and it is vertical between each straight line and the corresponding CCD. Since the straight line over the CCD point can be perceived, there are four points which are coplanar for each CCD. That is, the spatial point light, the CCD through the lens of the light point, the lens focus, and the projection point on the $X-Y$ plane. As a result, the three CCDs are extended into three planes, where only the spatial point light is communal. Note that the spatial point light positioning information is the intersection of the three planes. The positioning principle is shown in Figure 2(b).

4.1.2. Measurable Range on the Plane. The dynamic positioning system is verified using the following three cases:

Case 1: according to the principle of the spatial positioning system, every point on the $z=100 \mathrm{~mm}$ plane, and the range of $81 \times 81 \mathrm{~mm}^{2}$, the values are obtained on the spatial plane, and the points perceived by the sensor are the spatial points in the three CCD measurable ranges. As shown in Figure 4, the red area represents the measurable point, and the cyan area represents the undetectable point.

Case 2: in the determinable space $X=$ $[-2000,2000], Y=[-2000,2000], Z=6000$, the measurable spatial points are represented by red dots on the plane of $Z=6000 \mathrm{~mm}$ as shown in Figure 5 .

Case 3: measurable range of multilayer planes: in the case of the determined space $X=[-2000,2000]$, $Y=[-2000,2000], Z=6000$, the space plane is set from $Z=100 \mathrm{~mm}$ to $Z=6000 \mathrm{~mm}$, defining the measurable range of each spatial point be $500 \mathrm{~mm}$ as the additional steps. Figure 6(a) shows the measurable range for each spatial layer. Similarly, Figure 6(b) describes the results due to reducing the addition steps as $10 \mathrm{~mm}$, i.e., the measurable range from $Z=$ $90 \mathrm{~mm}$ to $Z=300 \mathrm{~mm}$ on the $X-Y$ plane. Figure 6 shows that the measurable range of spatial points is presented from point, line to triangle, until the polygon, which is dynamically changing.

Similarly, it is obvious that the more precise the pace on the CCD is, the more perceptible the number of points could be sensed. Therefore, the spatial positioning system possesses a wider measurement range. From the current simulation results, the range of measurable points in each spatial layer is still $Y$-axis symmetry, and the distribution profile of each layer point is from one point to the triangle, until the polygon measurement interval. Note that the schematic diagram of the dynamic measurement device is symmetry on the $Y$-axis as shown in Figure 2(a). According to the principle of the $3 \mathrm{D}$ photoelectric sensor, the projection from the spatial point source is also symmetry on each CCD plane. 
Input:

The initial state $x(0)$, measurement $z^{1}(0), \ldots, z^{L}(0)$, estimated state $\widehat{x}_{0 \mid 0}$, and error covariance $P_{0 \mid 0}$.

Output:

The local state estimation $\hat{x}_{\text {ASS }}^{1}(k \mid k), \ldots, \hat{x}_{\text {ASS }}^{L}(k \mid k)$ and the fused state estimation $\hat{x}(k \mid k)$.

(1) Perceive the measurements $z^{1}(k), \ldots, z^{L}(k)$ from sensor data packets with timestamp at the current time instant $k$, which are transmitted over the networked systems.

(2) Judge the received valid signals using the ASS scheme. The reorganized measurement is denoted as $y^{i}(t)$ for the $i$-th subsystem.

(3) Probe the filtering parameters $\widehat{C}_{\mathrm{ASS}, t}^{i}, K_{\mathrm{ASS}, t}^{i}, \widehat{A}_{\mathrm{ASS}, t}^{i}$, and $L_{\mathrm{ASS}, t}^{i}$ according to Theorem 2.

(4) Solve the filter $\widehat{x}_{\mathrm{ASS}}^{i}(t \mid t)$ and predictor $\widehat{x}_{\mathrm{ASS}}^{i}(t+1 \mid t)$ in equations (8) and (9).

(5) Obtain the weighted fusion estimation $\hat{x}(t \mid t)$ from equation (25).

(6) Update $k=k+1$ at the next time instant. Return to Step 2.

(7) return $\widehat{x}_{\mathrm{ASS}}^{i}(t \mid t)$ and $\widehat{x}_{\mathrm{ASS}}^{i}(t+1 \mid t)$ in equations (8) and (9), respectively. $\bar{\Theta}_{\mathrm{ASS}}^{i}(t), \bar{\Sigma}_{\mathrm{ASS}}^{i}(t+1)$, and $P(t+1)$ from equations (19)-(21).

Algorithm 1: Distributed weighted fusion estimation using the ASS scheme.

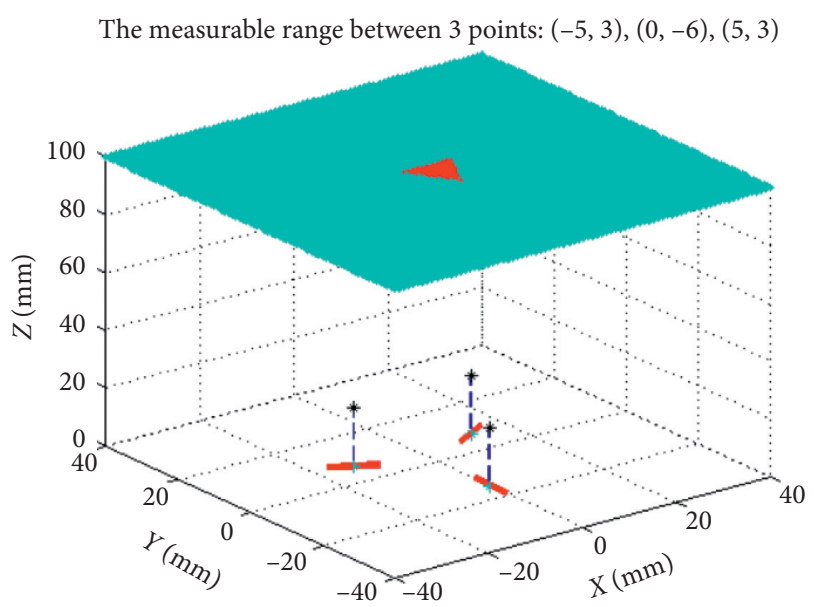

FIGURE 4: Measurable range on the $z=100 \mathrm{~mm}$ plane.

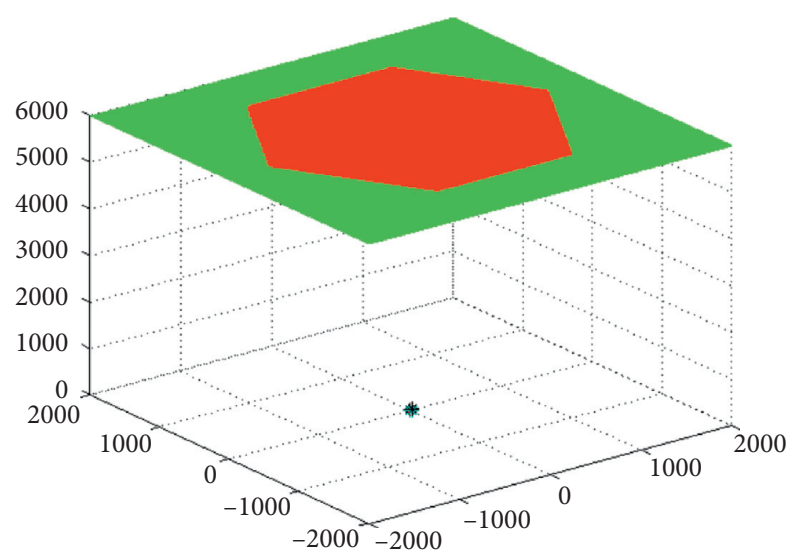

Figure 5: Measurable range on the $z=6000 \mathrm{~mm}$ plane.

4.2. Experimental Results. The effectiveness of the proposed distributed weighted fusion estimation strategy is validated by numerical examples. The dynamic positioning system for the intermittent measurement is considered as a distributed uncertain system [32, 37-39]:

$$
\begin{aligned}
x(k+1)= & \left(\left[\begin{array}{ccc}
0.9 & T & T^{2} / 2 \\
0 & 0.9 & T \\
0 & 0 & 0.9
\end{array}\right]+\mathscr{F}_{k} F_{k} E_{k}\right) x(k) \\
& +\left[\begin{array}{c}
T^{2} / 2 \\
T \\
1
\end{array}\right] w_{k}, \quad k=1,2, \ldots, \\
z^{i}(k)= & \left(C_{k}^{i}+\mathscr{H}_{k}^{i} F_{k} E_{k}^{i}\right) x(k)+v_{k}^{i}, \quad i=1,2,3, \\
w_{k}= & \eta_{k}, \\
v_{k}^{i}= & \zeta_{i} w_{k},
\end{aligned}
$$

where the sampling period is set as $T=0.1 \mathrm{~s}$. The maximum value of the transmission time delay is set as 5 sampling periods. The time-varying parameter uncertainty is satisfied as $F_{k}=\sin (0.6 k)$. System state $x(k)=\left(\begin{array}{lll}s_{k} & \dot{s}_{k} & \ddot{s}_{k}\end{array}\right)^{T}$ is used to represent the values of position, velocity, and acceleration for the target at $k T$. Set parameters as $\mathscr{F}_{k}=\left[\begin{array}{lll}0.1 & 0.1 & 0.1\end{array}\right]^{T}$, $E_{k}=\left[\begin{array}{lll}0.02 & 0.02 & 0.02\end{array}\right], \quad C_{k}^{1}=\left[\begin{array}{lll}0.6 & 0.8 & 1\end{array}\right], \quad C_{k}^{2}=$ $\left[\begin{array}{lll}1 & 0.8 & 0.5\end{array}\right], C_{k}^{3}=\left[\begin{array}{lll}0.3 & 1 & 0.7\end{array}\right]$, and $\mathscr{H}_{k}^{1}=\mathscr{H}_{k}^{2}=\mathscr{H}_{k}^{3}=0.8$. Define $\eta_{k} \in \mathbb{R}$ as zero-mean white noise with the variance $\sigma_{\eta}^{2}=0.09$. Considering the cross-correlation between the process and measurement noise, $\zeta_{i}$ in equation (28) is set as $\zeta_{1}=2, \zeta_{2}=0.8$, and $\zeta_{3}=1$, respectively. The covariance of the process noise in equation (28) is $w_{k}$ with the covariance $Q_{k}$ : Meanwhile, the covariance $R_{k}^{i, j}$ is represented as $R_{k}^{i, j}=\zeta_{k}^{i} \zeta_{k}^{j} Q_{k}$, and the mutual covariance is denoted as $S_{k}^{i}=$ $\zeta_{k}^{i} Q_{k}$ in equation (28).

The initial values are set as $\hat{x}(0 \mid 0)=\mu_{0}=E(x(0))=$ $\left[\begin{array}{lll}1 & 1 & 1\end{array}\right]^{T}$ and $P(0 \mid 0)=0.01 I_{3}$. For the numerical examples, 300 sampling points are selected, and the experimental results are verified based on 300 Monte Carlo simulation calculations. Comparing the traces of the estimated error covariance matrix, the results are shown in Figure 7 . The estimated state $\hat{x}(k \mid k)$ is validated by the improved robust finite-horizon Kalman filtering (IRFHKF) in [38], as well as the proposed distributed weighted fusion state estimation using an adaptive signal selection scheme (DWFASS). First of all, taking into account the transmission with delay-free from Figure $7(\mathrm{a})$, the upper boundary of the estimation error 


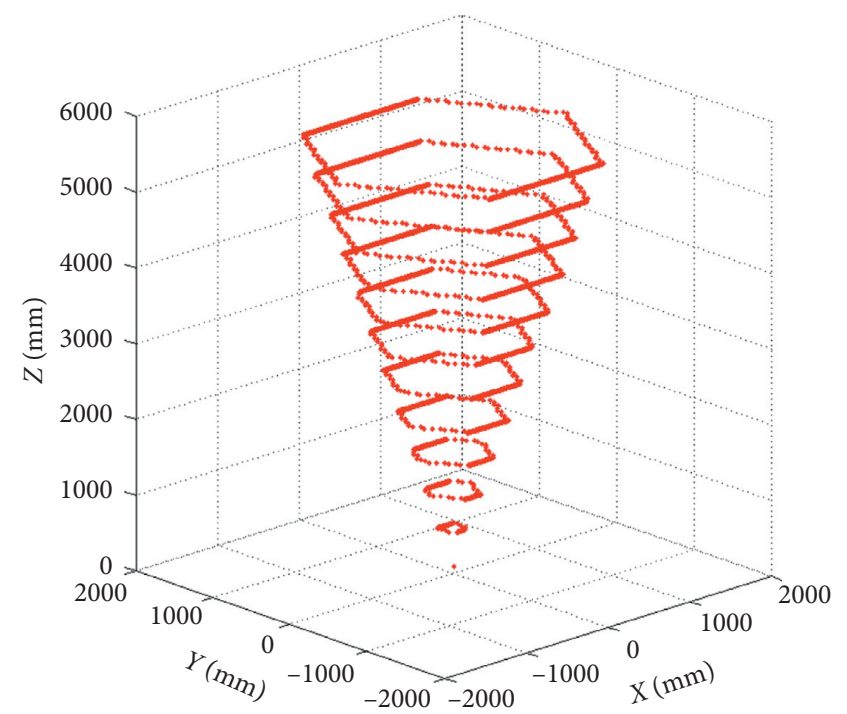

(a)

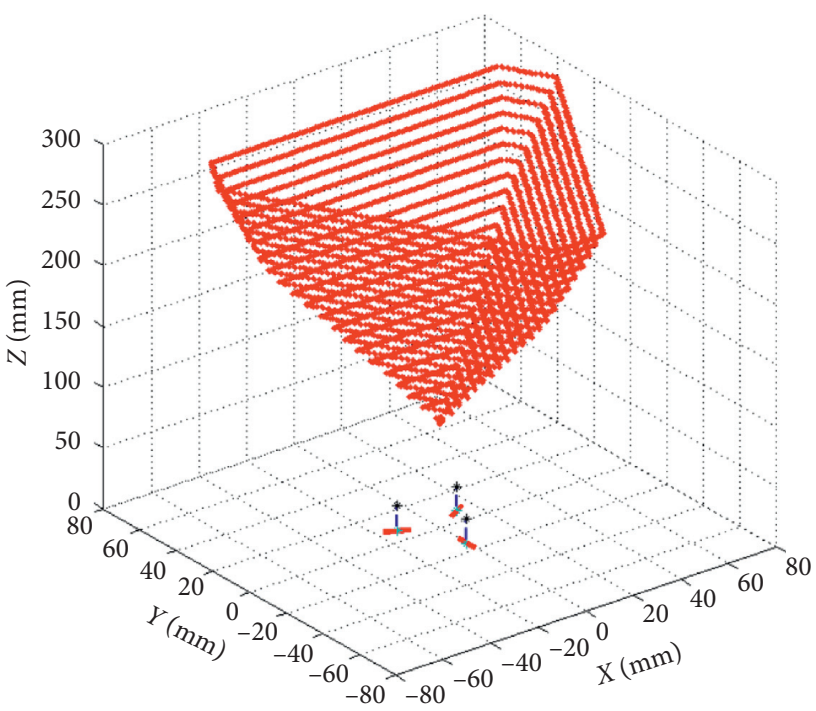

(b)

Figure 6: Measurable range of the sensor multilayer plane. (a) $Z=\{100,600, \ldots, 6000\}$. (b) $Z=\{90,100, \ldots, 300\}$.

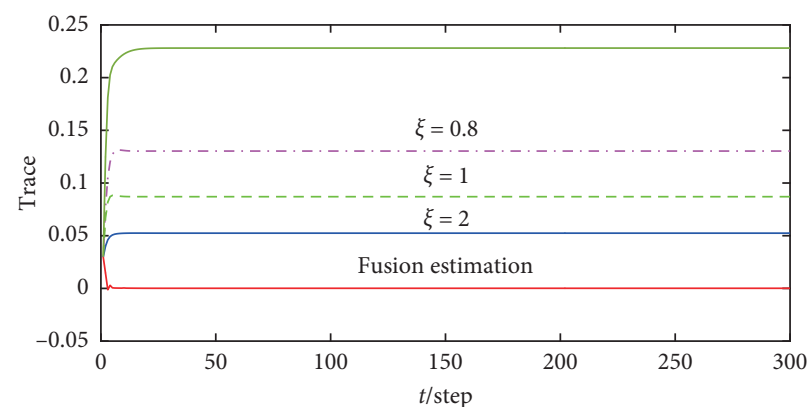

— DWFASS for sensor 1 DWFASS for fusion estimation

...- DWFASS for sensor 2 IRFHKF

- - - DWFASS for sensor 3

(a)

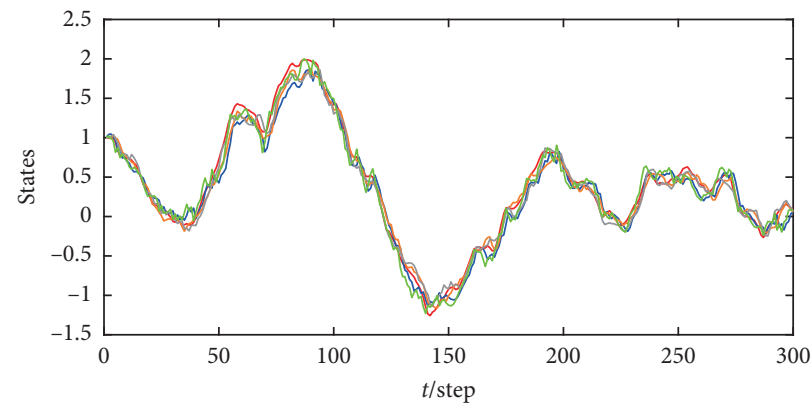

$\begin{array}{ll}\text { - Actual state of velocity } & \quad \text { DWFASS for sensor } 3 \\ \text { DWFASS for sensor } 1 & \text { IRFHKF } \\ \text { DWFASS for sensor } 2 & \end{array}$

(c)

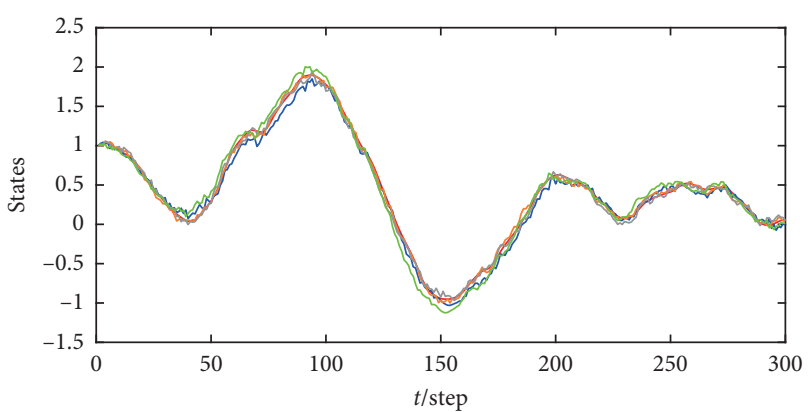

— Actual state of position $\quad$ DWFASS for sensor 3
DWFASS for sensor 1
DWFASS for sensor 2

(b)

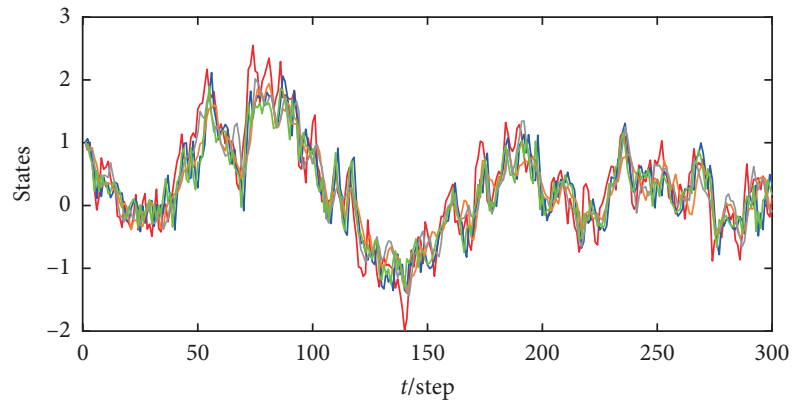

- Actual state of acceleration - DWFASS for sensor 3
DWFASS for sensor 1
DWFASS for sensor 2

(d)

FIgURE 7: Continued. 


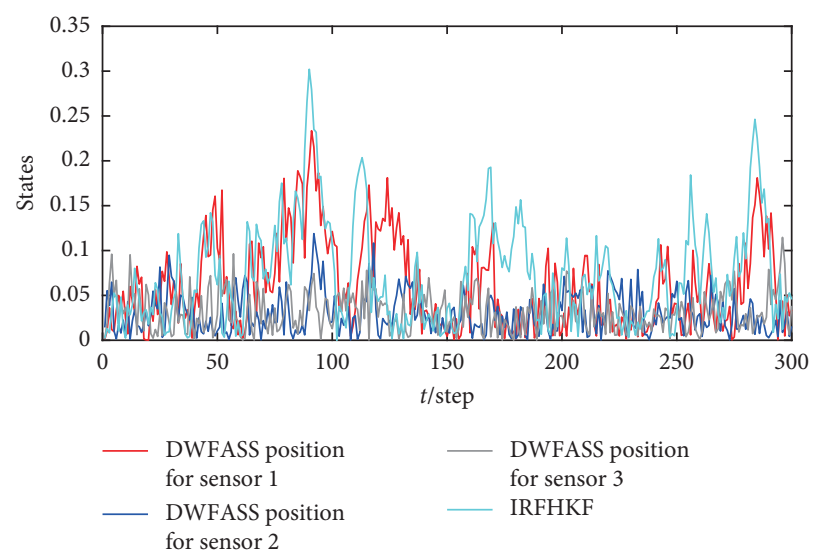

(e)

FIGURE 7: Comparing DWFASS and IRFHKF methods: (a) trace for the upper boundary, (b) comparison of the estimated position state, (c) comparison of the estimated velocity state, (d) comparison of the estimated acceleration state, and (e) comparison of MAEs for the position.

covariance matrix is small compared with the IRFHKF method. It is worth noting that the upper boundary of the minimum value for the fused cross-covariance matrix is smaller than the upper boundary for the other covariance matrices, since the weighted fusion criterion is the optimized again in the sense of the minimal variance. Comparing the two methods shown in Figures $7(\mathrm{~b})-7(\mathrm{~d})$, the proposed method is more suitable for detecting the optimal filtering parameters. Therefore, the designed estimator is appropriate for the uncertainty in model parameters as well as external disturbances, such as random transmission time delays and lost and disordered packets.

In addition, to further analyze the performance of the proposed DWFASS method, the maximum absolute errors (MAE) [42] are obtained by

$$
\text { MAE }=\left|x(k)-\widehat{x}_{\text {ASS }}^{i}(k \mid k)\right| .
$$

On the one hand, the root mean square error (RMSE) [43] between the measured and estimated values is defined as

$$
\mathrm{RMSE}=\sqrt{\frac{1}{M} \sum_{k=1}^{M}\left(x(k)-\hat{x}_{\mathrm{ASS}}^{i}(k \mid k)\right)^{2}},
$$

where $M$ represents the number of the measured points and $x(k)$ and $\hat{x}_{\mathrm{ASS}}^{i}(k \mid k)$ represent the measured and estimated states at the current sampling points $k$, respectively.

Figure $7(\mathrm{e})$ shows the MAEs comparing DWFASS and IRFHKF methods; meanwhile, the RMSEs are demonstrated in Table 1. Considering the cross-correlation for the noise, the dynamic tracking trajectory is closer to the actual state value if the noise is more correlated.

Next, for the distributed networked systems, the processing effect of each subsystem is different, which is determined by the cross-correlation for the system parameters and uncertain disturbances. Note that if the process noise is more cross-correlated with the measurement noise, the estimation performance is more accurate.
TABLE 1: Comparing RMSEs between DWFASS and IRFHKF.

\begin{tabular}{lccc}
\hline Methods & Position & Velocity & Acceleration \\
\hline Sensor 1 & 0.0469 & 0.0906 & 0.3182 \\
Sensor 2 & 0.0402 & 0.0697 & 0.3120 \\
Sensor 3 & 0.0426 & 0.0765 & 0.3140 \\
IRFHKF & 0.0681 & 0.0980 & 0.3831 \\
\hline
\end{tabular}

Moreover, to further illustrate the effectiveness of the DWFASS method, the distributed weighted fusion results for state estimation are shown in Figure 8. The results of state estimation are calculated from Theorems 2 and 3, and the method of the robust finite-horizon filtering is studied using the ASS scheme, as well as the distributed weighted fusion estimation criteria.

Figure 8 shows that the ASS scheme is able to actively discard data packets owing to the random transmission time delays and lost and disordered packets. At the same time, Table 2 enumerates the RMSEs of each sensor. Therefore, the distributed weighted fusion estimation uses the one-step prediction compensation for better performance and computational efficiency in target tracking.

4.3. Numerical Simulation Analysis. According to the simulation results, the dynamic tracking results are compared by the method of recombination error covariance. The comparative results show that the proposed estimation instrument has better estimation accuracy, especially in the case of communication constraints with multistep random delays and lost and disordered network induction packets. Because the true estimation error covariance is lower than the upper boundary of the probe, the proposed distributed fusion estimation method has better performance in the phenomenon of network induction, including the ability to closely track the state of the system. 


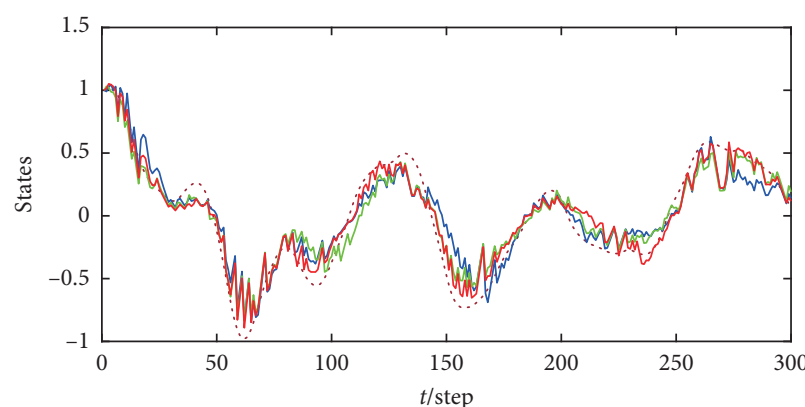

.... Actual state of position __ Estimated state of sensor 2

Estimated state of sensor $1 \_$Estimated state of sensor 3

(a)

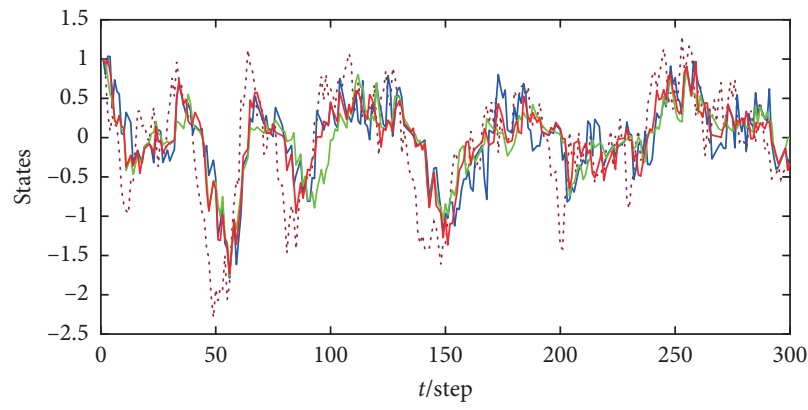

.... Actual state of acceleration __ Estimated state of sensor 2 _ Estimated state of sensor $1 \_$Estimated state of sensor 3

(c)

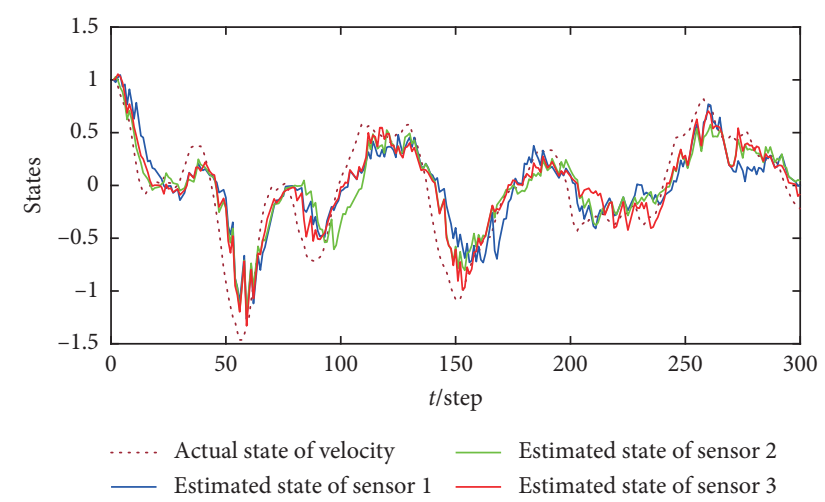

(b)

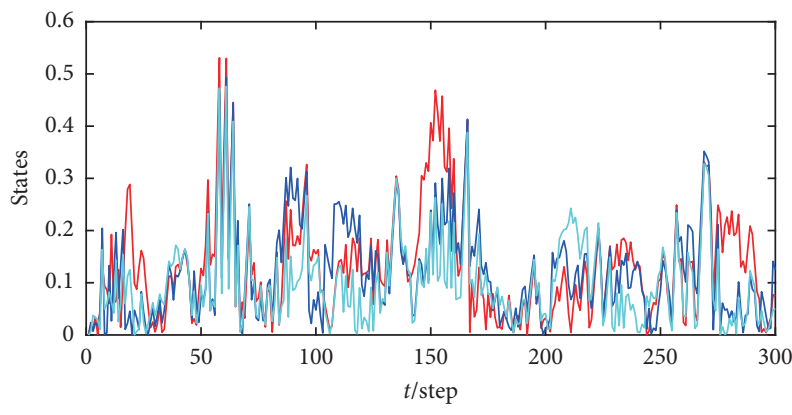

_ Estimated state of sensor 1 Estimated state of sensor 2 Estimated state of sensor 3

(d)

FIGURE 8: Comparison of distributed weighted fusion estimation results: (a) estimated position state, (b) estimated velocity state, (c) estimated acceleration state, and (d) comparison of MAEs for the position.

TABLE 2: Comparing RMSEs of each sensor.

\begin{tabular}{lccc}
\hline Methods & Position & Velocity & Acceleration \\
\hline Sensor 1 & 0.1658 & 0.2749 & 0.5745 \\
Sensor 2 & 0.1508 & 0.2553 & 0.5464 \\
Sensor 3 & 0.1273 & 0.2128 & 0.4909 \\
\hline
\end{tabular}

\section{Conclusion}

This paper, facing the spatial positioning principle of the linear CCD, introduces the workflow of the $3 \mathrm{D}$ photoelectric sensor dynamic positioning system and simulates the spatial positioning effect of the proposed DWFASS method. According to the measured spatial target, the trajectory of its motion is tracked and estimated, and a kind of distributed uncertain system with network-inducing factors is studied. In order to process the lost and disordered data packets by the random transmission time delays, the system is modelled based on the adaptive signal selection scheme. In order to overcome the influence of limited communication capacity and reduce the computational burden, this paper adopted the onestep prediction compensation strategy and proposed the optimal state estimation method to compensate for the missing packets. At the same time, the finite-horizontal filtering strategy is designed, and the real estimated error variance obtained by the DWFASS method is less than its upper boundary. Then, the distributed estimation method based on filtering is introduced, which introduces the uniform expression of the weighted fusion standard. Therefore, the distributed fusion estimation had higher estimation accuracy than that of each local subsystem. The numerical simulation is demonstrated using the spatial target positioning system containing three sensor devices, and the results show that the proposed distributed weighted fusion estimation has better tracking performance. To better process the data required for positioning and improve the accuracy of positioning, this paper has optimized the performance of positioning algorithms. The proposed method can be used in various motion control scenarios such as target tracking, trajectory tracking, path following, and formation control of AUVs subject to uncertainties and constraints. In addition, the future work will further focus on following some known distributions for the time delays or packet loss to further enhance the state estimation, such as the Gaussian or binary distribution based on the known bandwidth of the network.

\section{Data Availability}

The data used to support the findings of this study are included within the proposed information fusion estimation approach in the article. The data from numerical simulations 
are available from the established system model and the given initial values.

\section{Conflicts of Interest}

The authors declare that there are no conflicts of interest regarding the publication of this paper.

\section{Acknowledgments}

This work was supported by Natural Science Foundation of China (61903172, 61872170, 61903174, 61877065, 61873117, 61673200, 61771231), the Major Basic Research Project of Natural Science Foundation of Shandong Province of China (ZR2018ZC0438) and the Key Research and Development Program of YantaiCity of China (2019XDHZ085).

\section{References}

[1] Z. Zhang and Y. Dong, "Temperature forecasting via convolutional recurrent neural networks based on time-series data," Complexity, vol. 2020, Article ID 3536572, 8 pages, 2020.

[2] M.-F. Ge, C.-D. Liang, X.-S. Zhan, C.-Y. Chen, G. Xu, and J. Chen, "Multiple time-varying formation of networked heterogeneous robotic systems via estimator-based hierarchical cooperative algorithms," Complexity, vol. 2020, Article ID 8357428, 18 pages, 2020.

[3] A. Aydin, "Applications of artificial intelligence techniques to enhance sustainability of industry 4.0: design of an artificial neural network model as dynamic behavior optimizer of robotic arms," Complexity, vol. 2020, Article ID 8564140, 10 pages, 2020.

[4] X. Tang, K. Liu, X. Wang, F. Gao, J. Macro, and W. D. Widanage, "Model migration neural network for predicting battery aging trajectories," IEEE Transactions on Transportation Electrification, vol. 6, no. 2, pp. 363-374, 2020.

[5] C. D. Makavita, S. G. Jayasinghe, H. D. Nguyen, and D. Ranmuthugala, "Experimental study of command governor adaptive control for unmanned underwater vehicles," IEEE Transactions on Control Systems Technology, vol. 27, no. 1, pp. 332-345, 2019.

[6] H. Wu, X. Mei, X. Chen, J. Li, J. Wang, and P. Mohapatra, “A novel cooperative localization algorithm using enhanced particle filter technique in maritime search and rescue wireless sensor network," ISA Transactions, vol. 78, pp. 39-46, 2018.

[7] X. Tang, K. Liu, X. Wang, B. Liu, F. Gao, and W. D. Widanage, "Real-time aging trajectory prediction using a base modeloriented gradient-correction particle filter for lithium-ion batteries," Journal of Power Sources, vol. 440, Article ID 227118, 2019.

[8] Z. Zheng, Y. Huang, L. Xie, and B. Zhu, "Adaptive trajectory tracking control of a fully actuated surface vessel with asymmetrically constrained input and output," IEEE Transactions on Control Systems Technology, vol. 26, no. 5, pp. 1851-1859, 2018.

[9] K. Liu, Y. Li, X. Hu, M. Lucu, and W. D. Widanage, "Gaussian process regression with automatic relevance determination kernel for calendar aging prediction of lithium-ion batteries," IEEE Transactions on Industrial Informatics, vol. 16, no. 6, pp. 3767-3777, 2020.

[10] K. Liu, X. Hu, Z. Wei, Y. Li, and Y. Jiang, "Modified Gaussian process regression models for cyclic capacity prediction of lithium-ion batteries," IEEE Transactions on Transportation Electrification, vol. 5, no. 4, pp. 1225-1236, 2019.

[11] Z. Peng, J. Wang, and J. Wang, "Constrained control of autonomous underwater vehicles based on command optimization and disturbance estimation," IEEE Transactions on Industrial Electronics, vol. 66, no. 5, pp. 3627-3635, 2019.

[12] L. Liu, A. Yang, W. Zhou, X. Tu, G. Wang, and H. Wang, "Event-based finite horizon state estimation for stochastic systems with network-induced phenomena," Transactions of the Institute of Measurement and Control, vol. 41, no. 6, pp. 1580-1589, 2019.

[13] L. Liu, A. Yang, W. Zhou, W. Naeem, G. Wang, and H. Wang, "Modelling and estimation for uncertain systems with transmission delays, packet dropouts, and out-of-order packets," Complexity, vol. 2018, Article ID 7531547, 15 pages, 2018.

[14] J. Du, X. Hu, M. Krstić, and Y. Sun, "Dynamic positioning of ships with unknown parameters and disturbances," Control Engineering Practice, vol. 76, pp. 22-30, 2018.

[15] A. H. Brodtkorb, S. A. Værnø, A. R. Teel, A. J. Sørensen, and R. Skjetne, "Hybrid controller concept for dynamic positioning of marine vessels with experimental results," Automatica, vol. 93, pp. 489-497, 2018.

[16] E. Melek, T. T. Gokhan, A. W. Philip, and E. Seniz, "Marine measurement and real-time control systems with case studies," Ocean Engineering, vol. 159, pp. 457-469, 2018.

[17] G. Zhang, C. Huang, X. Zhang, and W. Zhang, "Practical constrained dynamic positioning control for uncertain ship through the minimal learning parameter technique," IET Control Theory \& Applications, vol. 12, no. 18, pp. 2526-2533, 2018.

[18] H. H. M. Weerts, P. M. J. Van den Hof, and A. G. Dankers, "Prediction error identification of linear dynamic networks with rank-reduced noise," Automatica, vol. 98, pp. 256-268, 2018.

[19] C. Xu, C. Xu, C. Wu et al., "A novel self-adapting filter based navigation algorithm for autonomous underwater vehicles," Ocean Engineering, vol. 187, Article ID 106146, 2019.

[20] M. Sato and M. Toda, "Adaptive algorithms of tuning and switching Kalman and $H_{\infty}$ filters and their application to estimation of ship oscillation with time-varying frequencies," IEEE Transactions on Industrial Electronics, vol. 67, no. 1, pp. 501-511, 2020.

[21] J. Ye, M. Godjevac, S. Baldi, and H. Hopman, "Joint estimation of vessel position and mooring stiffness during offshore crane operations," Automation in Construction, vol. 101, pp. 218-226, 2019.

[22] Z. Chen, B. Qin, M. Sun, and Q. Sun, "Q-learning-based parameters adaptive algorithm for active disturbance rejection control and its application to ship course control," Neurocomputing, 2019, In press.

[23] J. Brouwer, J. Tukker, Y. Klinkenberg, and M. van Rijsbergen, "Random uncertainty of statistical moments in testing: Mean," Ocean Engineering, vol. 182, pp. 563-576, 2019.

[24] M. Zhu, W. Sun, A. Hahn, Y. Wen, C. Xiao, and W. Tao, "Adaptive modeling of maritime autonomous surface ships with uncertainty using a weighted LS-SVR robust to outliers," Ocean Engineering, vol. 200, p. 107053, 2020.

[25] T. I. Fossen and J. P. Strand, "Passive nonlinear observer design for ships using lyapunov methods: full-scale experiments with a supply vessel," Automatica, vol. 35, no. 1 , pp. 3-16, 1999. 
[26] X. Ge and Q.-L. Han, "Distributed event-triggered $H_{\infty}$ filtering over sensor networks with communication delays," Information Sciences, vol. 291, pp. 128-142, 2015.

[27] X. Zhong, A. Mohammadi, A. B. Premkumar, and A. Asif, "A distributed particle filtering approach for multiple acoustic source tracking using an acoustic vector sensor network," Signal Processing, vol. 108, pp. 589-603, 2015.

[28] D. Li, S. Kar, J. M. F. Moura, H. V. Poor, and S. Cui, "Distributed Kalman filtering over massive data sets: analysis through large deviations of random Riccati equations," IEEE Transactions on Information Theory, vol. 61, no. 3, pp. 13511372, 2015.

[29] K. Liu, K. Li, Q. Peng, Y. Guo, and L. Zhang, "Data-driven hybrid internal temperature estimation approach for battery thermal management," Complexity, vol. 2018, Article ID 9642892, p. 15, 2018.

[30] K. Liu, Y. Shang, Q. Ouyang, and W. D. Widanage, “A datadriven approach with uncertainty quantification for predicting future capacities and remaining useful life of lithiumion battery," IEEE Transactions on Industrial Electronics, vol. 1, 2020.

[31] O. L. V. Costa and G. R. A. M. Benites, "Robust mode-independent filtering for discrete-time Markov jump linear systems with multiplicative noises," International Journal of Control, vol. 86, no. 5, pp. 779-793, 2013.

[32] J. Feng, Z. Wang, and M. Zeng, "Distributed weighted robust Kalman filter fusion for uncertain systems with autocorrelated and cross-correlated noises," Information Fusion, vol. 14, no. 1, pp. 78-86, 2013.

[33] L. Yan, X. Rong Li, Y. Xia, and M. Fu, "Optimal sequential and distributed fusion for state estimation in cross-correlated noise," Automatica, vol. 49, no. 12, pp. 3607-3612, 2013.

[34] A. Keshavarz-Mohammadiyan and H. Khaloozadeh, "Consensus-based distributed unscented target tracking in wireless sensor networks with state-dependent noise," Signal Processing, vol. 144, pp. 283-295, 2018.

[35] L. Liu, A. Yang, X. Tu, M. Fei, and W. Naeem, "Distributed weighted fusion estimation for uncertain networked systems with transmission time-delay and cross-correlated noises," Neurocomputing, vol. 270, pp. 54-65, 2017.

[36] S. Wang, H. Fang, and X. Tian, "Minimum variance estimation for linear uncertain systems with one-step correlated noises and incomplete measurements," Digital Signal Processing, vol. 49, pp. 126-136, 2016.

[37] T. Tian, S. Sun, and N. Li, "Multi-sensor information fusion estimators for stochastic uncertain systems with correlated noises," Information Fusion, vol. 27, pp. 126-137, 2016.

[38] H. Rezaei, R. Mahboobi Esfanjani, and M. H. Sedaaghi, "Improved robust finite-horizon Kalman filtering for uncertain networked time-varying systems," Information Sciences, vol. 293, pp. 263-274, 2015.

[39] S. Wang, H. Fang, and X. Tian, "Recursive estimation for nonlinear stochastic systems with multi-step transmission delays, multiple packet dropouts and correlated noises," Signal Processing, vol. 115, pp. 164-175, 2015.

[40] S.-L. Sun and Z.-L. Deng, "Multi-sensor optimal information fusion Kalman filter," Automatica, vol. 40, no. 6, pp. 10171023, 2004.

[41] B. Chen, W.-A. Zhang, and L. Yu, "Distributed fusion estimation with missing measurements, random transmission delays and packet dropouts," IEEE Transactions on Automatic Control, vol. 59, no. 7, pp. 1961-1967, 2014.
[42] J. Kim, J.-K. Min, and K. Shim, "Efficient two-dimensional $\mathrm{Haar}^{+}$synopsis construction for the maximum absolute error measure," The VLDB Journal, vol. 28, no. 5, pp. 675-701, 2019.

[43] M. Ćalasan, S. H. E. A. Aleem, and A. F. Zobaa, "On the root mean square error (RMSE) calculation for parameter estimation of photovoltaic models: a novel exact analytical solution based on Lambert W function," Energy Conversion and Management, vol. 210, Article ID 112716, 2020. 